\title{
Disfunção craniocervicomandibular com alteração do VIII par craniano em distrófico muscular: relato de caso
}

Craniocervicomandibular dysfunction with alteration of the VIII cranial pair in muscular dystrophic: case report Disfunción craneocervicomandibular con alteración del par craneal VIII en distrofia muscular: reporte de caso Karoliny Fernandes TAVARES ${ }^{1}$ Lioney Nobre CABRAL ${ }^{2}$

${ }^{1}$ Curso de Graduação em Odontologia pela Universidade do Estado do Amazonas, UEA, 69065-001 Manaus - AM, Brasil

${ }^{2}$ Doutor em Biotecnologia pela Universidade Federal do Amazonas, UFAM, Professor Adjunto, Curso de Odontologia, Universidade do Estado do Amazonas, UEA, 69065-001 Manaus - AM, Brasil

\section{Resumo}

A disfunção craniocervicomandibular é uma condição comum, acredita-se que dois terços da população sofrerão dor cervical em algum momento da vida. Caracterizada por dor cervical; limitação da amplitude dos movimentos da região; ruídos articulares e tensão dos músculos da região cervical. Anatomicamente, a região cervical é a base e o suporte das estruturas da cabeça e pescoço, e por causa de sua proximidade e inervações comuns, as desordens da coluna cervical podem se manifestar com dores na região orofacial. Quando essa disfunção acomete uma pessoa com quadro distrófico muscular grave, tende a ter uma severidade muito maior. Este trabalho tem como objetivo avaliar a disfunção craniocervicomandibular em paciente portador de distrofia muscular compatível com Duchenne. Paciente 22 anos, gênero masculino compareceu a Policlínica Odontológica da Universidade do Estado do Amazonas, na clínica de Estomatologia queixando-se de incomodo na mandíbula, com ruído e dor. O mesmo relatou sentir constantes dores de cabeça, plenitude auricular e vertigem. Em seguida aos exames clínicos, avaliação das desordens temporomandibular e avaliação muscular, evidenciou-se o diagnóstico de Disfunção Craniocervicomandibular com Vestibulococleopatia. Deu-se início ao tratamento associada a fisioterapia a fim de condicionar um ambiente para o uso de placa miorrelaxante.

Descritores: Cervicalgia; Distrofia Muscular de Duchenne; Dor Facial; Modalidades de Fisioterapia.

\section{Abstract}

Craniocervicomandibular dysfunction is a common condition, it is believed that two thirds of the population will suffer cervical pain at some point in life. Characterized by cervical pain; limitation of the range of motion of the region; joint noises and muscle tension in the cervical region. Anatomically, the cervical region is the base and support of head and neck structures, and because of its proximity and common innervations, cervical spine disorders may manifest with pain in the orofacial region. When this dysfunction affects a person with severe muscular dystrophic condition, it tends to be much more severe. This study aims to evaluate craniocervicomandibular dysfunction in a patient with Duchenne compatible muscular dystrophy. A 22 -year-old male patient attended the Dentistry Polyclinic of the Amazonas State University at the Stomatology Clinic complaining of jaw discomfort, noise and pain. He reported experiencing constant headaches, ear fullness and dizziness. Following clinical examinations, evaluation of temporomandibular disorders and muscle evaluation, the diagnosis of Craniocervicomandibular Dysfunction with Vestibulococleopathy was evidenced. Physiotherapy-associated treatment was initiated to condition an environment for the use of myorelaxant plaque.

Descriptors: Neck Pain; Muscular Dystrophy, Duchenne; Facial Pain; Physical Therapy Modalities.

\section{Resumen}

La disfunción craneocervicomandibular es una condición común, se cree que dos tercios de la población experimentarán dolor cervical en algún momento de la vida. Caracterizado por dolor cervical; limitación del rango de movimiento de la región; ruidos articulares y tensión muscular en la región cervical. Anatómicamente, la región cervical es la base y el soporte de las estructuras de la cabeza y el cuello, y debido a su proximidad e inervaciones comunes, los trastornos de la columna cervical pueden manifestarse con dolor en la región orofacial. Cuando esta disfunción afecta a una persona con condición distrófica muscular severa, tiende a ser mucho más severa. Este estudio tiene como objetivo evaluar la disfunción craneocervicomandibular en un paciente con distrofia muscular compatible con Duchenne. Un paciente masculino de 22 años asistió al Policlínico de Odontología de la Universidad Estatal de Amazonas en la Clínica de Estomatología quejándose de molestias en la mandíbula, ruido y dolor. Informó experimentar dolores de cabeza constantes, llenura de oídos y mareos. Después de los exámenes clínicos, la evaluación de los trastornos temporomandibulares y la evaluación muscular, se evidenció el diagnóstico de disfunción craneocervicomandibular con vestibulococleopatía. El tratamiento asociado con la fisioterapia se inició para acondicionar un ambiente para el uso de la placa miorrelajante.

Descriptores: Dolor de Cuello; Distrofia Muscular de Duchenne; Dolor Facial; Modalidades de Fisioterapia.

INTRODUÇÃO

A desordem craniocervicomandibular é um termo utilizado para descrever uma condição que abrange uma variedade de problemas clínicos associados a alterações funcionais do sistema mastigatório, da articulação temporomandibular (ATM), da coluna cervical e estrutura adjacentes ${ }^{1}$.

A Distrofia Muscular de Duchenne é uma doença neuromuscular hereditária progressiva, mais comum nas duas primeiras décadas de vida, com manifestações clínicas geralmente vistas a partir do segundo ano. Ligada ao cromossomo $\mathrm{X}$, afeta predominantemente meninos cursando com hipotonia muscular e fraqueza muscular precoces. Leva às alterações

\section{mais diversas, esqueléticas e funcionais ${ }^{2}$.}

Em relação a fisiopatologia da Distrofia Muscular, sabe-se que há uma deficiência ou ausência de distrofina na superfície da membrana da célula muscular (Sarcolema). A distrofina é uma proteína fundamental para o funcionamento do sarcolema, sendo esta considerada a lesão inicial nas fibras musculares distróficas na Distrofia Muscular ${ }^{3}$.

Em pacientes distróficos é possível notar o aumento da concentração de cálcio no interior da célula, desencadeando uma contração máxima do músculo, provocando um déficit metabólico local, este metaboliza gás carbônico no lugar de oxigênio, ou seja, ocorre uma 
anaerobiose, com produção mínima de energia, 2 ATP e ácido láctico, essa falta de fonte de energia acomete os sarcômeros que não possuem ATP suficiente para ativar a bomba de Cálcio, impedindo seu retorno para o retículo sarcoplasmático e procedendo uma contração muscular máxima ${ }^{3}$ Levando ao estimulo do sistema nervoso central por meio de fibras aferentes moduladas no complexo de interneurônios. Logo, ativando outras áreas neuronais, promovendo então uma dor referida, concorrendo para alterações miofasciais que levam a alterações a distância ${ }^{4}$.

Anatomicamente, a região cervical é a base e o suporte das estruturas da cabeça e pescoço, e por causa de sua proximidade e inervações comuns, as desordens da coluna cervical podem se manifestar com dores na região orofacial ${ }^{5}$. Uma postura inadequada pode levar a dor e alteração funcional de vários sistemas. Como exemplo, pode-se citar a protrusão da cabeça, a qual pode afetar a função dos músculos anteriores do pescoço, como esternocleidomastoideo, escaleno, supra e infra-hiódeos ${ }^{6}$. O conhecimento das disfunções musculares maxilo mandibulares e sua relação com estruturas contíguas e toda a economia orgânica é um fator de grande interesse para todas as especialidades da Odontologia?.

A disfunção craniocervicomandibular é uma condição comum. Contudo, quando essa disfunção acomete uma pessoa com quadro distrófico muscular, tende a ter uma severidade muito maior. O tratamento é multidisciplinar com médico, dentista e fisioterapeuta. O presente relato de caso, demonstra a importância da terapia de escolha para tratar a disfunção craniocervicomandibular associada a fisioterapia a fim de condicionar um ambiente para o uso da placa miorrelaxante, com mudanças propceptivas, e alivio do quadro de dor, tonturas e cefaléia, melhorando a qualidade de vida de um paciente que apresentava disfunção crâniocervicomandibular com sintomatologia agravada, provocada pela sua condição muscular patológica.

\section{CASO CLÍNICO}

Paciente 22 anos, gênero masculino, leucoderma, residente da cidade de Manaus, compareceu a Policlínica Odontológica da Universidade do Estado do Amazonas, na clínica de Estomatologia tendo como queixa principal "Sinto incomodo na minha mandíbula, às vezes faz ruído e às vezes dói" $O$ mesmo relatou sentir constantes dores de cabeça, presença de plenitude auricular e vertigem. É portador de Distrofia muscular congênita combatível com Duchenne, uma doença degenerativa de origem genética (ligada ao cromossomo $\mathrm{X}$ ) diagnosticado logo ao nascimento (Figura 1).

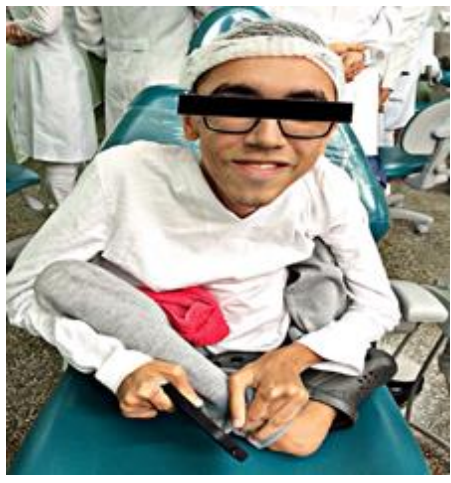

Figura 1: Perfil clínico do paciente portador de Distrofia Muscular Congênita Combatível com Duchenne.

Em anamnese, paciente relatou sentir essa dor há mais de quatro anos, tendo buscado tratamento médico com especialista otorrinolaringologista devido sentir dores constantes no ouvido e sensação de plenitude auricular. No exame foi descartada a hipótese de qualquer infecção auditiva, encaminhando-o a um dentista especializado em tratar disfunção temporomandibular; relatando ainda ter problemas respiratório e gástrico. Em sua história médica pregressa paciente informou ter nascido cianótico e apresentando características de distrofia muscular. Após exame clínico e anamnese, foi realizado avaliação das desordens temporomandibular por meio de alguns procedimentos como mensurações utilizando especímetro e os resultados obtidos foram: 1) Avaliação da abertura máxima de boca, obtendo $24 \mathrm{~mm}$ (Figura 2); 2) Interferência mandibular, apresentan- do desvio na abertura com retorno a linha média (Figura 3); 3) Em protrusão, não ocorreu desvio, afirmando possuir presença de zumbido e plenitude auricular na orelha direita e presença de vertigem, além de fortes dores de cabeça.

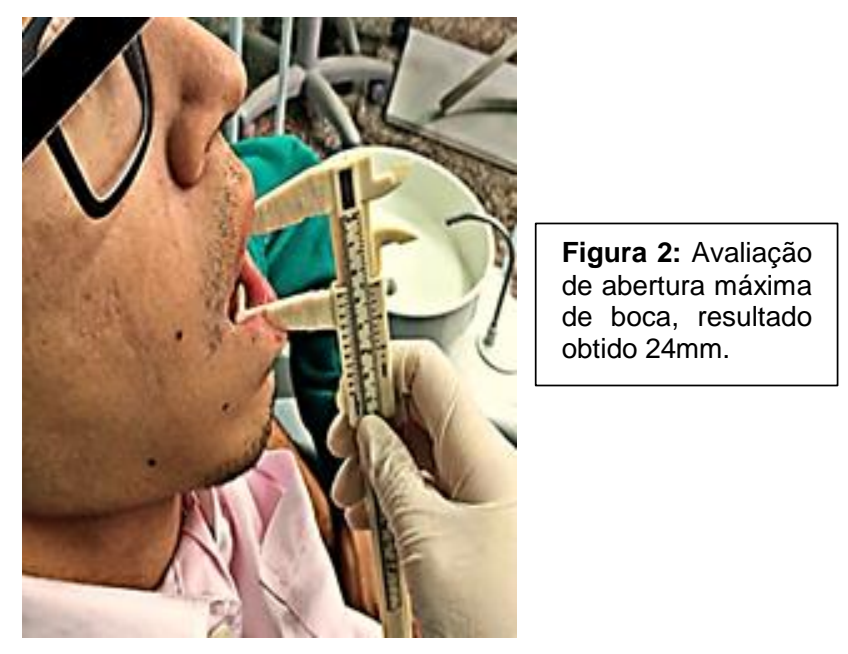




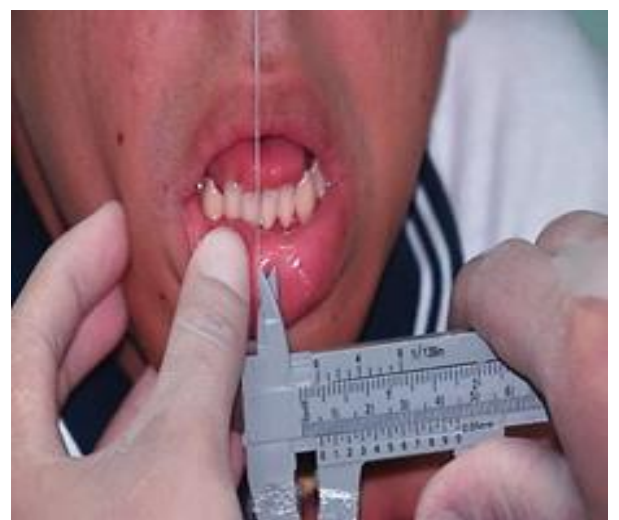

Figura 3: Interferência mandibular onde apresentou desvio na abertura com retorno a linha média.

Foi realizado avaliação muscular inicial, com palpação dos músculos temporal, masseter e esternocleidomastoideo, afim de encontrar áreas com tônus muscular alterados, espasmos e pontos gatilhos (trigger points). Durante palpação do músculo temporal paciente relatou dor em região de feixe anterior, médio e posterior, assim como apresentou dor na porção cranial da parte superficial do masseter e dor na porção caudal; na região profunda do masseter, paciente apresentou reação de fuga em resposta a palpação do local, no esternocleidomastoideo, também apresentou dolorimento, sendo constatado que o lado direito do paciente era o mais afetado com maior sintomatologia dolorosa (Figura 4) e (Figura 5). Em seguida aos exames clínicos, avaliação das desordens temporomandibular e avaliação muscular, evidenciou-se o diagnóstico de Disfunção Craniocervicomandibular com Vestibulococleopatia. Prontamente foi realizada revisão da literatura com intuíto de estabelecer uma relação entre a Distrofia muscular de Duchenne e a Disfunção Craniocervicomandibular, concluindo que, sua doença de base atua como uma predisposição para 0 desenvolvimento da disfunção craniocervicomandibular de uma forma mais acentuada e dolorosa.

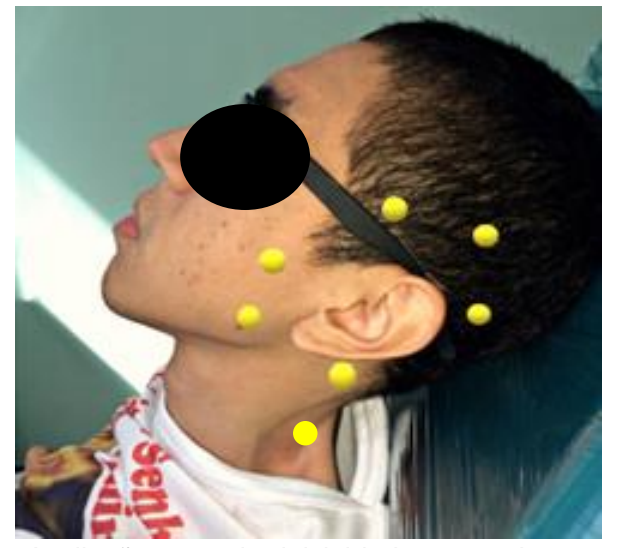

Figura 4: Avaliação muscular inicial lado esquerdo apresentando dolorimento em músculo temporal, masseter e esternocleidomastoideo, ilustrado por bolas amarelas nos locais de dor.

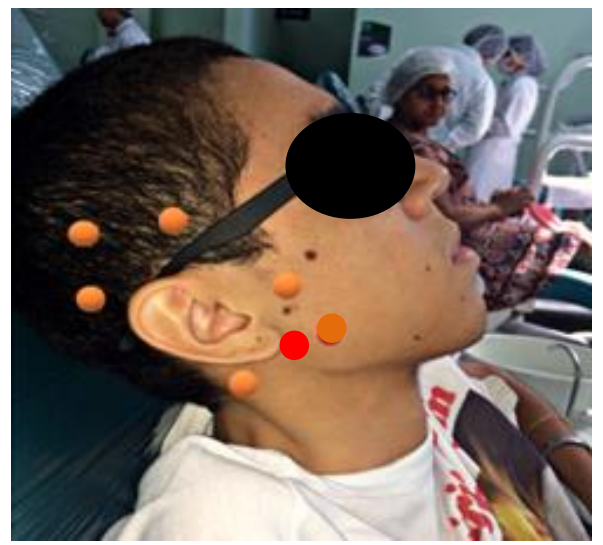

Figura 5: Avaliação muscular lado direito ilustrado com bolas laranjas nos locais de dor mais intensa (pontos gatilhos) e bola vermelha em masseter profundo onde paciente apresentou reação de fuga.

O paciente apresenta um perfil clínico de atrofia muscular dos membros e escoliose severa, concorrendo para uma postura que prejudique seu equilíbrio corporal levando a alterações no sistema estomatognatico, e havendo envolvimento da musculatura orofacial. Diante dos estudos obtidos e ao diagnóstico de Disfunção Craniocervicomandibular deu-se início ao tratamento associada a fisioterapia a fim de condicionar um ambiente para o uso de placa miorrelaxante. Foi realizado primeiramente moldagem da arcada superior e inferior do paciente para confecção de placa interoclusal. Em seguida à moldagem, foi realizada a tomada do arco facial para registrar os movimentos mandibulares e a oclusão do paciente. Após uma semana, paciente retornou a Policlínica Odontológica da Universidade do Estado do Amazonas para instalação da placa miorrelaxante, recebendo informações referentes aos exercícios a ser realizado antes do uso da placa. A terapia consistia em exercícios mandibulares de abertura contra resistência realizado por 10 segundos 03 vezes ao dia. Exercício de fechamento contra resistência também a ser realizado 10 segundos 3 vezes por dia. Foi indicada a termoterapia que consiste na aplicação terapêutica de frio e calor no local como compressas de água quente ou gelada de acordo com o clima para aliviar dores musculares e articulares e promover o relaxamento muscular a fim de condicionar um ambiente adequado para uso da placa (Figuras 6 e 7). Após uma semana de uso da placa miorrelaxante, paciente retornou para proservação, apresentando melhora expressiva com ausência de dor, vertigem, cefaléia e plenitude auricular. Foi realizada avaliação muscular final para observação dos pontos gatilhos em comparação a primeira avaliação muscular, constatando-se ausência de dor em músculo, temporal, masseter e esternocleidomastoideo como consta na 
(Figuras 8 e 9). Após um ano de uso da placa interoclusal e acompanhamento, o paciente retornou para confecção de uma nova placa, devido à quebra do dispositivo, durante esse período sem uso da placa, paciente relatou sintomatologia dolorosa nos músculos da face. Foi realizada no momento da consulta avaliação muscular com palpação dos músculos Temporal, Masseter e Esternocleidomastoideo confirmando a sintomatologia dolorosa.

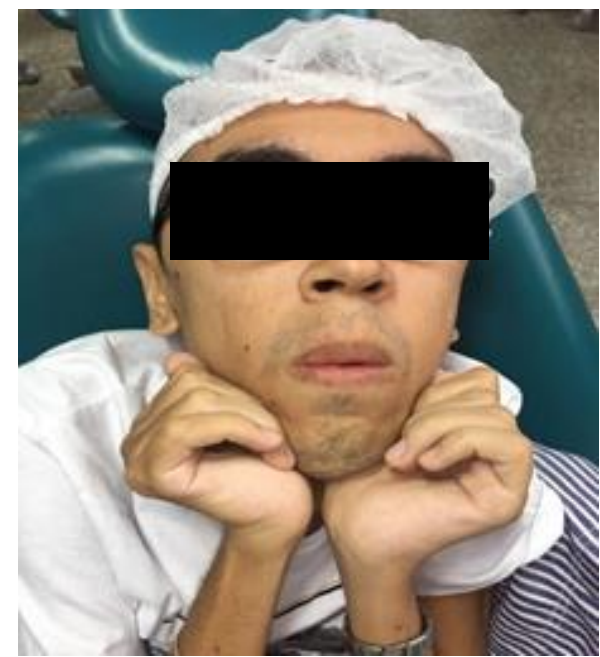

Figura 6: Paciente realizando exercício de abertura contra a resistência.

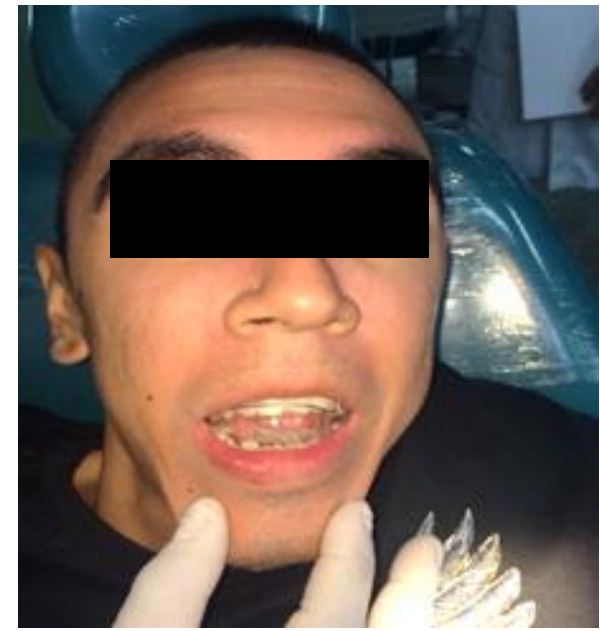

Figura 7: Instalação de placa miorrelaxante no paciente.

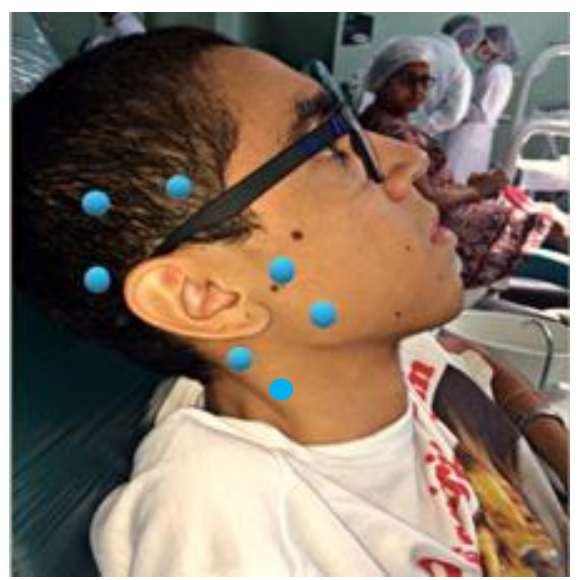

Figura 8: Ausência de pontos gatilhos lado esquerdo ilustrado com bolas azuis.

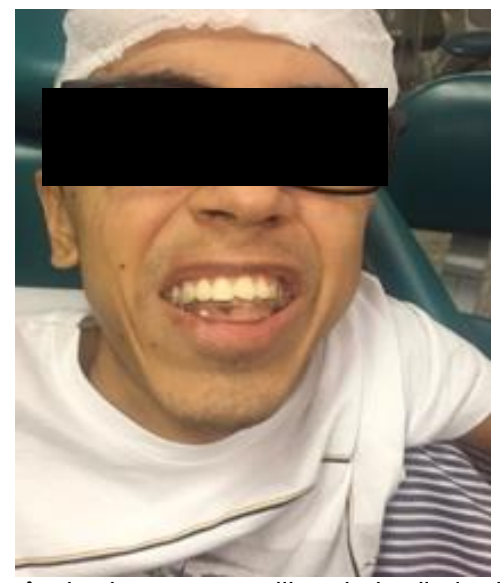

Figura 9: Ausência de pontos gatilhos lado direito ilustrado com bolas azuis.

Orientou-se ao paciente que durante a espera da nova placa ele continuasse com os exercícios fisioterapêuticos afim de aliviar a dor. Após duas semanas paciente retornou para instalação do novo dispositivo interoclusal sendo realizado nova reavaliação muscular.

Os resultados obtidos foram: Músculo temporal feixe anterior, médio e posterior, lados direito e esquerdo se apresentaram com ausência de dor. Músculo Masseter também apresentou ausência de dor em região proximal, corpo, distal, porém em região profunda ocorreu dor leve do lado esquerdo e direito. Esternocleidomastoideo houve presença de dor na porção mastoidea e clavicular. Posteriormente a uma semana com a placa instalada paciente encontrava-se sem dor novamente. (Figura10)

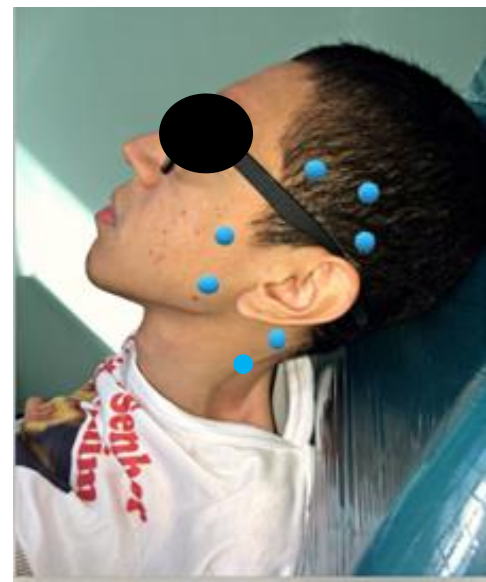

Figura 10: Após um ano paciente com nova placa miorrelaxante instalada.

DISCUSSÃO

A desordem craniocervicomandibular é um termo utilizado para descrever uma condição que abrange uma variedade de problemas clínicos associados a alterações funcionais do sistema mastigatório, da articulação temporomandibular (ATM), da coluna cervical e estrutura adjacentes ${ }^{1}$. 
As disfunções temporo- mandibulares referem-se a um conjunto de condições que afetam os músculos da mastigação e/ou a articulação temporomandibular. Quando esta condição abrange também problemas clínicos na região cervical, utiliza-se o termo disfunção craniocervicomandibular. Além de comprometer funções próprias do sistema estomatognático, as alterações nas estruturas da ATM podem trazer sintomatologia auditiva associada devido à grande proximidade anatômica e funcional entre os componentes da orelha e ATM ${ }^{8}$

Segundo Gama et al. $^{9}$ as disfunções craniocervicomandibular miogênicas (DCCM) são consideradas desordens que apresentam várias sugestões etiológicas com relatos de dor. A etiologia da DCCM é controversa e considerada como multifatorial, incluindo fatores como as desarmonias esqueléticas, más oclusões, desvios de forma nos componentes da ATM, traumas, bruxismo, parafunção muscular, doenças degenerativas da articulação e fatores emocionais. Nas DCCMs miogênicas podem ocorrer estalos ou crepitações ao abrir e fechar a boca, mioespasmos, dor na musculatura mastigatória e região cervical ${ }^{9}$. No caso em questão e de acordo aos achados na literatura os sintomas referidos pelo paciente foi de fortes dores na musculatura mastigatória, crepitação ao abrir e fechar a boca, assim como apresentava doença muscular degenerativa.

Existe uma correlação dos sintomas e sinais (dor orofaciais, ruídos na ATM, cefaleia, bruxismo, dificuldade de abrir a boca e anteriorização da cabeça com lateralização para o lado) de DTM com a coluna cervical, devido à cadeia muscular posterior sofrer uma possível tensão, podendo gerar uma alteração postural ocasionando hiperlordose cervical e retração mandibular, fazendo com que ocorra um desequilíbrio, podendo assim afetar o sistema estomatognatico ${ }^{10}$.

Para Halmova et al. ${ }^{11}$ a posição incorreta do corpo, compressão da raiz espinhal isométrica prolongada, alterações degenerativas nos tendões e ligamentos, também são considerados fatores etiológicos do transtorno assim como a formação de dor miofascial na cabeça e pescoço é significativamente influenciado pela hiperextensão da coluna vertebral (C1-C3) e flexão de sua parte inferior (C4-C7). A hiperlordose da coluna implica na posição anterior da cabeça e a hipercifose da coluna leva à posição anterior dos ombros ${ }^{11}$. Silva, Queiroz e Serrano afirmam que todos os seres humanos necessitam de uma postura corporal estável e equilibrada como apoio, no qual os movimentos voluntários e coordenados podem ser iniciados como parte das funções naturais. Qualquer posição que aumente o estresse nas articulações pode ser chamada "má postura", estes problemas podem induzir disfunções do crânio, tais como fadiga dos músculos cervicais, surgimento de pontos gatilhos (trigger points) e indução de dor cranial $^{12}$. No relato de caso apresentado o paciente é portador de Distrofia Muscular Congênita compatível com Duchenne, tendo como característica patológica atrofias musculares severas, com escoliose grave, apresentando sérias complicações posturais.

De acordo com os autores Fernandes e Barbosa $^{13}$ já que a disfunção temporomandibular apresenta etiologia multifatorial, tanto o diagnóstico quanto o tratamento tornam-se mais complexos, devendo o cirurgião-dentista utilizar todos os recursos disponíveis para relacionar causa e efeito, fazendo uso de terapias como ajuste oclusal, reabilitação protética, dispositivos oclusais, dentre outros ${ }^{13}$. No presente relato de caso optou-se pela confecção de placa miorrelaxante concomitante aos exercios fisioterapêuticos aderindo à literatura apresentada.

Moraes et al. $^{2}$ relatam que a Distrofia Muscular de Duchenne é uma doença neuromuscular hereditária progressiva mais comum nas duas primeiras décadas de vida com manifestações clínicas geralmente vistas a partir do segundo ano. Ligada ao cromossomo $X$ que afeta predominantemente meninos cursando com hipotonia muscular e fraqueza muscular precoces. O diagnóstico é feito através da biologia molecular, dosagem de creatino fosfoquinase e biopsia muscular e deve ser feito o mais precocemente possível para permitir o uso de terapias que melhorem a qualidade de vida do paciente ${ }^{2}$. Destoando da literatura quanto às primeiras manifestações clínicas, no caso em questão paciente apresentou logo ao nascimento, porém concordando com o gênero mais acometido pela patologia, assim como ao diagnóstico realizado por meio de biopsia muscular.

$\mathrm{Cruz}^{14}$ em sua pesquisa afirma que de acordo com a revisão sistemática do ano de 2017, estima-se que a Distrofia Muscular de Duchenne afeta 15,9 a 19,5 homens por 100.000 nascidos vivos no mundo, considerado como distrofia a nível muscular mais frequente e grave. Dentro das repercussões sistêmicas, a literatura mostra que é afetado o sistema estomatognático, observando ainda uma alta prevalência de cárie (> $60 \%$ ) e más oclusões (86\%) em comparação com a população saudáveis ${ }^{14}$. Concordando com a literatura no 
referido caso clínico o paciente apresentou sistema estomatognático afetado, assim como más oclusões do tipo mordida aberta, e cálculo supra gengival.

Moraes et al. $^{2}$ e Santos et al. ${ }^{15}$ relatam em seus estudos que a doença é causada por uma alteração no gene da distrofina que está localizado no braço curto do cromossomo $X$, numa região denominada $\mathrm{Xp} 2^{11}$. Sua ausência acarreta alterações no sarcolema que sofre pequenos rompimentos provocando pequenos furos que aumentam a passagem de cálcio para dentro da célula levando a uma necrose segmentar da fibra e perda da sua propriedade contrátil $^{2,15}$. Um fator importante salientado no presente caso concertando com a literatura é o músculo do paciente que devido sua doença encontra-se com ausência da proteína distrofina, convergindo para uma contração muscular constante, logo, desencadeando pontos gatilhos, estes encontrados na avaliação muscular inicial, que levam estímulos ao sistema nervoso central por meio de fibras aferentes moduladas no complexo de interneurônios, resultando em ativação de outras áreas neuronais, promovendo dor referida com alterações a distância. Isso explica a plenitude auricular quando não havia nenhuma infecção, mas sim uma alteração muscular no Masseter, dores de cabeças constantes e tonturas proveniente de alteração do músculo temporal e esternocleidomastoideo.

As terapias propostas apresentaram efeitos positivos na redução da sintomatologia dolorosa, com total remissão da dor à palpação nos músculos temporal, masseter e esternocleidomastoideo, ausência dos sintomas de plenitude auricular e vertigem. $O$ tratamento não é apenas odontológico, exige um trabalho multidisciplinar. O uso de placa estabilizadora concomitante a fisioterapia, trabalhando-se exercícios de abertura contra resistência, com intuito de condicionar um ambiente para o uso da placa miorrelaxante. Trazendo qualidade de vida ao paciente que apresentava uma disfunção craniocervicomandibular acentuada com sintomatologia dolorosa agravada devido sua doença de base.

CONCLUSÃO

$\mathrm{Na}$ literatura revisada estudou-se a fisiopatologia da Distrofia Muscular de Duchenne com intuíto de estabelecer uma relação com a Disfunção Craniocervicomandibular concluindo que, sua doença de base atua como uma predisposição para 0 desenvolvimento da disfunção craniocervicomandibular. O paciente apresenta um perfil clínico de atrofia muscular e escoliose severa, concorrendo para uma postura que prejudique seu equilíbrio corporal levando a alterações no sistema estomatognático, e havendo envolvimento da musculatura orofacial. Um fator importante analisado é o músculo do paciente que devido sua doença encontra-se com ausência de uma proteína muito importante, convergindo para uma contração muscular constante, desencadeando pontos gatilhos, promovendo dor referida. Isso explica a plenitude auricular quando não havia nenhuma infecção, mas sim uma alteração muscular no Masseter, dores de cabeças constantes e tonturas proveniente de alteração do músculo temporal e esternocleidomastoideo. As terapias propostas associando 0 uso de placa miorrelaxante e exercícios fisioterapêuticos de abertura contra resistência como terapia manual tiveram resultados positivos, com objetivo de aliviar a dor e melhorar a função do paciente que apresentou evoluções significativas nos sintomas relatados na primeira consulta.

\section{REFERÊNCIAS}

1. Armijo-Olivo S, Rappoport $\mathrm{K}$, Fuentes J, Gadotti IC, Major PW, Warren S, Thie NM, Magee DJ. Head and cervical posture in patients with temporomandibular disorders. J Orofac Pain. 2011;25(3):199-209.

2. Moraes FM, Fernandes RSC, Acosta EM. Distrofia Muscular de Duchenne: relato de caso. Rev-Cient-FMC. 2011; 6(2):11-15.

3. Mokri B, Engel AG. Duchenne dystrophy: electron microscopic findings pointing to a basic or early abnormality in the plasma membrane of the muscle fibre. Neurology. 1975;25(12): 111-20.

4. Schmidt KC, Briesemeister M, Ries LGK. Changes in Mandibular and Cervical Motor Control of Children With Cerebral Palsy. Rev CEFAC. 2014;16(1):228-35.

5. Moreira AB, Morete M, Cordon F, Malezan WR. Disfunção Temporomandibular e dor Cervical: Comorbidades? Rev Aten Saúde. 2016;14(48):94-8.

6. Bigaton DR, Silvério KCA, Berni KCS, Distefano G, Forti F, Guirro RRJ. Postura craniocervical em mulheres disfônicas. Rev Soc Bras Fonoaudiol. 2010;15(3):329-34.

7. Martins BLA, Braga DRLM, Cabral LN. Disfunção massetérica e sialoadenite de refluxo: relato de caso. Arch Health Invest. 2019;8(1):43-7.

8. Godinho GV, Cabral LN. Disfunção craniocervicomandibular e alterações vestibulococleares: revisão de literatura. Arch Health Invest. 2019;8(8):405-12.

9. Gama BF, Barros FAM, Cardoso MBSC, Soares MA. Efeito da laserterapia baixa 
potência em pacientes com disfunção crânio cérvico-mandibular miogênica - análise através da biofotogrametria da biofotogrametria estudo duplo cego. Persp Online. 2015;17(5):36-46.

10. Souza I, Oliveira R, Jacques $H$, Barradas N, Luzes R. A Relação de Coluna Cervical Com a Disfunção Temporomandibular e Seus Principais Sinais e Sintomas. Alumni UNIABEU. 2017;5(9):4-9.

11. Halmova K, Holly D, Stanko P. The influence of cranio-cervical rehabilitation in patients with myofascial temporomandibular pain disorders. Bratisl Lek Listy. 2017;118(11):710-13.

12. Silva F, Queiroz A, Serrano K. Alteraciones posturales y su repercusión en el sistema estomatognatico. Acta odontol venez. 2008;46(4):517-22.

13. Fernandes Neto AJ, Barbosa GA. Placas oclusais. Uberlândia: Universidade Federal de Uberlândia (UFU). 2005. 19 p.

14. Cruz L, Tegtmeier C, Consiglio M, Barrionuevo L, Correa K. Características clínicas orales en pacientes con distrofia muscular de Duchenne. Estudio descriptivo. Rehabil Integral 2018;13 (2):59-65.

15. Silva MM, Santos E, Sardelich PL. Distrofia Muscular de Duchenne: repercussões pós treinamento muscular respiratório relato de caso. RUEP. 2016;13(32):84-91.

\section{CONFLITO DE INTERESSES}

Os autores declaram não haver conflitos de interesse

\section{AUTOR PARA CORRESPONDÊNCIA}

\section{Karoliny Fernandes Tavares}

Curso de Odontologia,

Universidade do Estado do Amazonas, UEA

69065-001 Manaus - AM, Brasil

E-mail:kft.odo@uea.edu.br 